\title{
New drug targets resistant tuberculosis
}

$\mathrm{T}$ he World Health Organization has taken the unprecedented step of releasing interim guidelines for the use of bedaquiline (Sirturo) to treat multidrug-resistant tuberculosis, although it hasn't yet undergone phase III trials. It is the first new drug to treat tuberculosis in more than 40 years.

The WHO released its guidelines June 13, after doctors had already started using the drug to treat adult patients with multidrug-resistant tuberculosis (MDRTB). Eight months ago, the United States Food and Drug Administration (FDA) approved the drug.

The guidelines list five conditions that must be in place before doctors use bedaquiline to treat patients with MDRTB. These include close monitoring; prohibitions on its use in pregnant women and children and caution in seniors and those infected with HIV; informed consent; the inclusion of the current TB regimen; and active monitoring and management of adverse events.

This new drug arrives at a time when patients with MDR-TB are in desperate need of more options. "TB is a life-threatening disease," the FDA stated when it approved the drug. "The development of resistance limits the therapeutic options available to treat patients with TB."

MDR-TB is believed to be on the rise. The World Health Organization estimates that there are up to a half-million new cases each year worldwide.

In Canada, bedaquiline is being used to treat only one patient with extensively drug-resistant TB, says Dr. Monica Avendaño, associate professor at the University of Toronto's Faculty of Medicine in Ontario. The patient is doing very well, she reports.

Avendaño, who obtained the drug through Health Canada's Special Access Program, said she is monitoring the patient closely but does not question the drug's safety. "If it had not been found to be safe, it wouldn't have been released," she says. "Without this

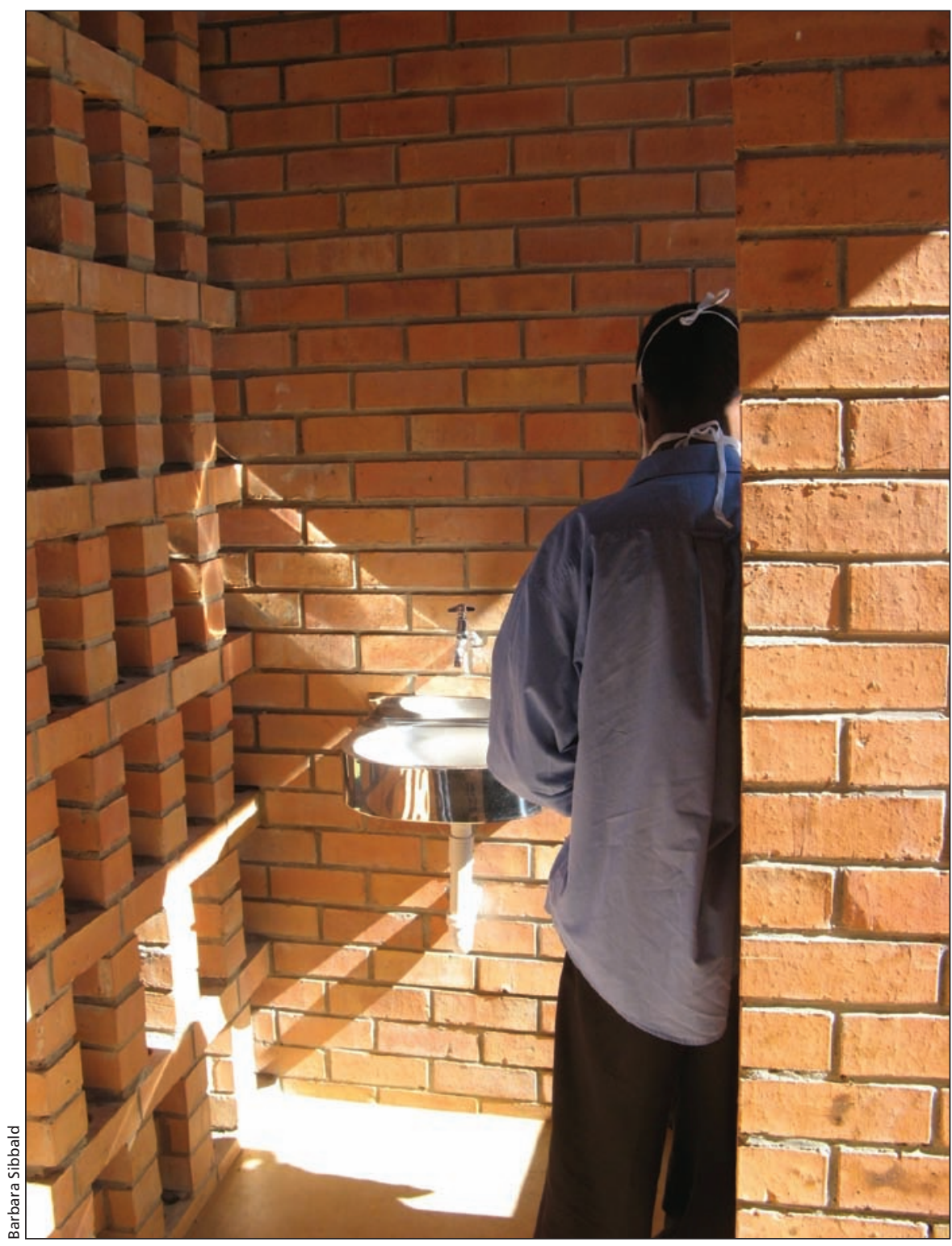

The World Health Organization has taken the unprecedented step of releasing interim guidelines for using bedaquiline (Sirturo) to treat multidrug-resistant tuberculosis, although it has not yet undergone phase III trials.

drug, the chances for the patient are not that good anyway."

Bedaquiline works differently than other drugs to combat Mycobacterium tuberculosis, the bacteria causing TB. It inhibits the mycobacterial ATP (adenosine 5'-triphosphate) synthase, a protein the bacterium needs to survive.

The drug is not intended to replace anything currently on the market, but will be used along with four other medications for patients with a multidrugresistant strain of tuberculosis. Standard practice currently is to combine multiple toxic drugs, which result in adverse effects similar in severity to those of chemotherapy.

Before the release of bedaquiline, the WHO recommended using four drugs concurrently, including injections for the 
first eight months and a total treatment length of 20 months. In 2009, only $48 \%$ of patients with MDR-TB were treated successfully. The hope is that bedaquiline will increase this percentage.

Bedaquiline is currently available in South Africa through a clinical access program that is treating about 10 patients, based on compassionate grounds. Applications to have the drug approved are underway in Thailand, India, South Africa and China.

A TB doctor with Médecins Sans Frontières in Khayelitsha, South Africa is supervising the treatment of three patients on the drug, and plans to obtain the drug for more patients. "People have been asking questions about what situations [bedaquiline] can be used in," says Dr. Jennifer Hughes. Before the drug is more widely released, Hughes believes doctors need additional information about drug interactions and how to work the medication into an existing TB regimen, especially for patients who are also HIV-positive.

The FDA approved bedaquiline in December 2012 through its accelerated approval process, which allows early approval of certain drugs that treat serious diseases without other good treatment options. Only phase IIb clinical trials have so far been completed. These trials examine the safety and efficacy of a drug in a smaller group of patients. Phase III trials test the way a drug performs in a larger population and more real-life situations.

Based on the trials, the drug's most common adverse effects include nausea, joint pain and headaches. The drug carries a warning from the FDA that it may affect the heart's electrical activity, causing QT prolongation, a potentially fatal event. The drug's label also notes there were more deaths in the bedaquiline group than the placebo group in the studies.

The WHO's support for bedaquiline may change pending the results of future trials, the organization says, warning there is little evidence so far for the drug's efficacy and safety.

A representative of Janssen Pharmaceutica, the unit of Johnson \& Johnson that developed the drug, said the company plans to make the drug more affordable in certain countries on a tiered system based on the country's burden of disease and its wealth. Amelia Wilkinson, CMAJ

CMAJ 2013. DOI:10.1503/cmaj.109-4540 